\title{
Simulation of the influence of surface chemical composition on internal gas flow at large Knudsen numbers
}

\author{
Alexander I. Ukhov ${ }^{*}$, Sergey F. Borisov ${ }^{1}$, Boris T. Porodnov ${ }^{2}$ \\ Ural Federal University, Yekaterinburg, Russia
}

\section{A R T I C L E I N F O}

\section{Article history:}

Received 25 November 2012

Received in revised form 5 May 2013

Accepted 6 May 2013

Available online 30 May 2013

\section{Keywords:}

Gas-surface interaction

Rarefied gas flow

Adsorbate

Auger spectroscopy

Numerical methods

\begin{abstract}
A B S T R A C T
On the basis of the developed model, an attempt to describe a rarefied gas flow in the cylindrical channel, whose surface chemical composition conforms with the real experimental conditions, has been made. During the modeling two cases are considered: the atomically clean silver channel surface and the surface fully covered with the adsorbate, which is simulated by oxygen according to the data of the Augerspectroscopic analysis. The gas-surface interaction process is based on the molecular dynamic principle. Thermal vibrations of the surface atoms are also considered. The obtained results of calculation are compared with experimental data.
\end{abstract}

Crown Copyright @ 2013 Published by Elsevier Ltd. All rights reserved.

\section{Introduction}

When the degree of gas rarefaction increases, the role of interface interaction and the efficiency of heat-and-mass transfer grow considerably as the intermolecular collisions become negligible compared to the molecular collisions with the surface. In the extreme case of the so-called free-molecular regime, the efficiency of heat-and-mass transfer in the "gas-solid body" system is fully determined by the processes occurring on the surface [1].

Until recently, the quantitative estimation of the influence of gas-surface interaction on heat-and-mass transfer represented a considerable problem. Particularly, the reviews containing the experimental data related to the efficiency of heat exchange between a rarefied gas and a solid body provide an extensive field of non-reproducible results [2]. Thus, for example, for the $\mathrm{He}-\mathrm{W}$ systems with similar gas and surface temperatures, different researchers obtain the values of energy accommodation coefficients that have a 20 -fold difference $(0.02$ and 0.4$)$. It means that, depending on the state of the surface, the heat fluxes for such system differ by 20 times in the free-molecular regime. More or less reliable reproducible data were obtained only for clean surfaces prepared according to the special techniques [3], as well as under the conditions that can be implemented on the molecular beam

\footnotetext{
* Corresponding author. Tel.: +7 9089224570.

E-mail addresses: sasha.ukhov@gmail.com (A.I. Ukhov), porodnov@dpt.ustu.ru (B.T. Porodnov).

1 Passed away during the process of preparation this paper.

2 Tel.: +7 9221151611.
}

scattering apparatuses. An impressive result is given in the paper [4], where the removal of surface contaminations results in the tangential momentum accommodation coefficient reaching the minimum value of $\sim 0.1$ for the $H e-A u$ system. For a "dirty" surface, the value of the tangential momentum accommodation coefficient is close to 1 . Thus, the mass flow for such system in the free-molecular regime can change up to 10 times depending of the state of the surface. It is obvious that the surface condition is of crucial importance in such experiments.

Now the gas-surface interaction problem becomes especially topical due to the development of a new area in the rarefied gas dynamics: Gas Micro Electro Mechanical Systems (GASMEMS). The examples of such systems can include micro-actuators, mass flow and temperature sensors, micro heat-exchangers for electronic component cooling. The fact that the reduction of device dimensions leads to the increase of the Knudsen number $K n=\lambda / D$ is the key issue that arises in the design process of such devices. Thus, the free-molecular regime is reached at higher gas pressures when the adsorption process becomes of primary importance.

The complexity of theoretical description of gas-surface interaction is connected with the lack of knowledge about the chemical and physical structure of the surface layers. The experimental studies show that the efficiency of energy and mass exchange between a solid surface and gas molecules is affected by multiple factors: these include gas and wall temperatures, gas pressure, type of gas molecules (monoatomic, polyatomic gasses or gas mixtures), chemical composition of the surface (adsorbed layers), as well as the surface roughness. An incident gas molecule can reflect back to the gas phase without exchanging energy with the surface, 


\section{Nomenclature}

Kn Knudesen number, rel. units

D characteristic length of the system, $m$

$r \quad$ radius of the channel, $m$

$l \quad$ length of the channel, $\mathrm{m}$

$F \quad$ interaction force, $\mathrm{N}$

$G \quad$ relative gas flow rate, rel. units

I ion current, rel. units

E energy of electrons, $\mathrm{eV}$

$N$ intensity of emission of Auger electrons, rel. units number of molecules

$L \quad$ exposure, $10^{-4}$ Pa s, relative length, rel. units

$T$ temperature, $\mathrm{K}$

$k \quad$ bondong force constant, $\mathrm{m}^{-1}$

$M \quad$ mass of the atom, $\mathrm{kg}$

W transmission probability

$U \quad$ potential

$x, y, z, \quad$ coordinates of particle

$X, Y, Z X^{\prime}, Y^{\prime}, Z^{\prime}$ cartesian axes in physical space

$v_{m} \quad$ most probable molecular speed in an equilibrium gas, $\mathrm{m} \mathrm{s}^{-1}$

a a constant

$\Delta t \quad$ integration time interval, $\mathrm{s}$

$R f() \quad$ random fraction between 0 and 1

$P \quad$ pressure, $\mathrm{Pa}$

$k_{b} \quad$ Boltzmann constant, $k=1.38065810^{-23} \mathrm{~J} \mathrm{~K}^{-1}$

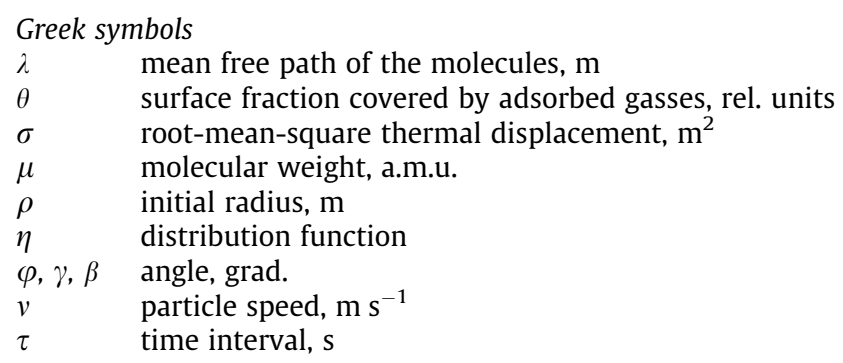

\section{Subscripts}

exp experimental

diff diffusely, diffuse

g gas

$S \quad$ surface

$0 \quad$ initial

gs gas-surface atomic pair

ga gas-adsorbate atomic pair

ss surface-surface atomic pair

sa surface-adsorbate atomic pair experience multiple collisions with the surface reaching the equilibrium with it. A particle can also be captured by the surface with the subsequent desorption caused by stochastic processes inside and on the surface of the solid body, or with the formation of a strong chemical bond.

The existing phenomenological structures, such as scattering kernels proposed by Maxwell [5], Cercignani-Lampis [6] and Epstein [7], have a mathematical basis and are artificial to a certain extent. Therefore, each given model has some limitations caused by the complexity of description of the processes occurring at the phase interface. One can make a conclusion that, presently, there is no a multi-purpose scattering kernel fully describing the process of interaction between the gas atoms and the solid body surface. Thus, a more detailed description of interaction requires creation of the models with a greater number of parameters, or movement to a "lower" level of the process description, where the physical and chemical structure of the surface, as well as the interatomic interaction potentials are simulated, using the techniques of molecular dynamics (MD).

Today, due to the growth of the computation power, the simulation based on the MD methods is a powerful tool both for studying individual interactions between gas atoms and a surface and for simulating flows of liquids and gasses in channels. Thus, for example, in the paper [8] the MD method was used for simulating the interaction between a monoatomic gas molecule and the molecules constituting a solid wall in order to study the characteristics of the reflected molecules at the solid surface. Recently, the socalled hybrid methods have become popular [9-12]. Their main idea is to use the MD methods to calculate the interaction of a gas atom with a surface, whereas the motion of gas molecules is simulated using the direct simulation Monte-Carlo. In the above examples of the hybrid methods, a gas particle interacts with surface atoms that are inside the so-called cut-off radius, which size is set based on the parameters of the interaction potential. Thus, the larger the cut-off radius, the more surface particles need to be taken into account when simulating the interaction, and the higher the computation accuracy. On the other hand, the reduction of the cut-off radius results in the decrease the computation time, but the simulation accuracy in this case notably drops. An example of a more computationally cost-effective method is given in the paper [13], where the vibration potential wall model is proposed. In this model, collisions occur between a particle and a part of a surface assumed as a rigid vibrating body. During the interaction, other particles of the surface are ignored, which allows reducing the computation time.

\section{Experimental background}

The growth of the processing power and the development of the computer simulation methods, in their turn, allow achieving new results. Particularly, the present paper attempts to describe the rarefied gas flow in a cylindrical channel, whose surface structure and chemical composition conforms to the real experimental conditions [14]. Let us provide a brief description of the experiment. A Pyrex glass cylindrical channel with radius $r=3.6 \mathrm{~mm}$ and a length $l=124 \mathrm{~mm}(L=l / r=34.4)$ with a metallic (considered $\mathrm{Ti}$ and $\mathrm{Ag}$ ) filament stretched along the axis is placed in an ultra-high vacuum system. The surface preparation process consists in the deposition of a metal film on the inner channel surface at the residual pressure of about $10^{-10} \mathrm{~Pa}$ by heating the respective metal filament. The surface chemical composition is monitored using the Augerspectroscopic analysis. The mass spectrum of residual gasses of the vacuum chamber (see Fig. 1) and the Auger spectrum derivatives of the channel surface (see Fig. 2) are used to indicate the formation of an adsorbed layer.

Gas flow rate $G_{\text {exp }}$ was measured by the technique of efflux from the vacuum chamber at pressure $P_{1}=10^{-8} \mathrm{~Pa}$ and temperature $T_{1}=300 \mathrm{~K}$ through the capillary into the high-vacuum chamber at pressure $P_{2}=10^{-10} \mathrm{~Pa}$ and temperature $T_{2}=300 \mathrm{~K}$.

The measurement results of the relative gas flow rate $G$ of the cylindrical channel in the free-molecular regime for different gasses $(\mathrm{He}, \mathrm{Ar}, \mathrm{Kr})$ are given in Fig. 3, where the left side of the graph corresponds to the freshly deposited $T i$ surface and the right side is 


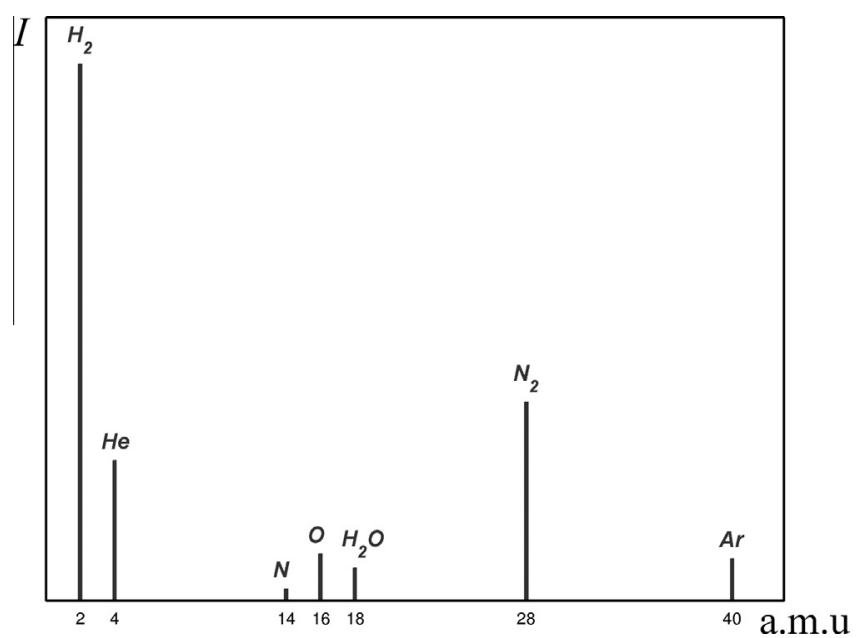

Fig. 1. Typical mass spectrum of residual gasses in the vacuum chamber [14].

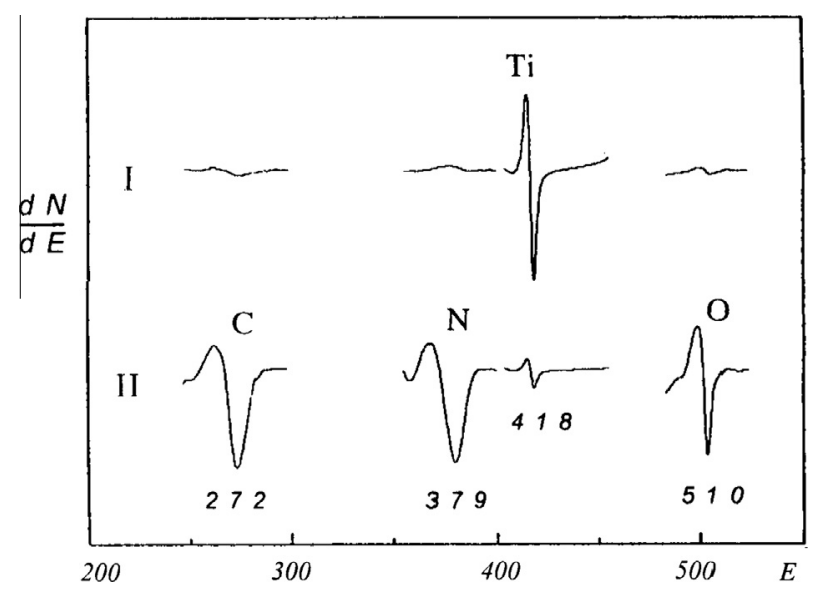

Fig. 2. Auger-spectrum derivatives for the "clean" surface (I) immediately after deposition of Ti, and the "dirty" surface (II) after prolonged exposure in the atmosphere of residual gasses of the vacuum chamber [14].

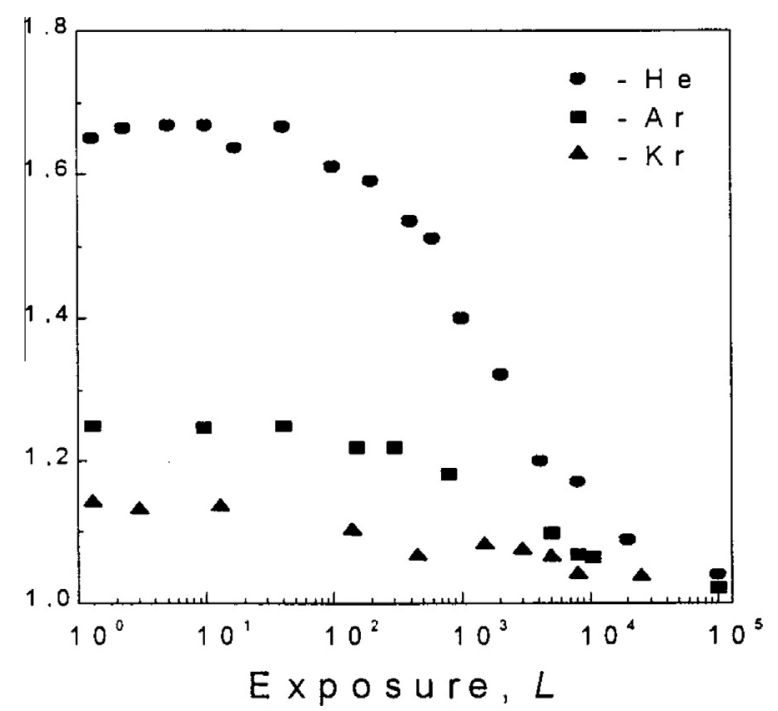

Fig. 3. Relative gas flow rate $G$ of the cylindrical channel in the free-molecular regime as a function of the oxygen exposure [14]. the surface fully covered with the adsorbate after prolonged exposure in the atmosphere of residual gasses of the vacuum chamber.

The $G$ value is determined as $G=G_{\text {exp }} / G_{\text {diff }}$, where $G_{\text {exp }}$ stands for the experimental gas flow rate and $G_{\text {diff }}$ is the calculated gas flow rate in the free-molecular regime for completely diffuse reflection for given $L=l / r$ ratio.

In the case of cylindrical channel the value of $G_{\text {diff }}$ for completely diffuse reflection can be calculated using (1) [15] if the transmission probability $W_{\text {diff }}$ for given $L$ is known.

$G_{\text {diff }}=r^{2} \sqrt{\frac{\pi m}{2 k_{b}}} W_{\text {diff }}\left(\frac{P_{1}}{\sqrt{T_{1}}}-\frac{P_{2}}{\sqrt{T_{2}}}\right)$

Transmission probability of the cylindrical channel in the case of completely diffuse reflection $W_{\text {diff }}$ for arbitrary $L$ can be calculated using (2) [15].

$$
\begin{aligned}
W_{\text {diff }}(L)= & 1+\frac{L^{2}}{4}-\frac{L}{4} \sqrt{L^{2}+4} \\
& -\frac{\left[\left(8-L^{2}\right) \sqrt{L^{2}+4}+L^{3}-16\right]^{2}}{72 L \sqrt{L^{2}+4}-288 \ln \left[L / 2+\sqrt{L^{2} / 4+1}\right]}
\end{aligned}
$$

Thus, in our case $W_{\text {diff }}(L=34.4)=0.068$.

The values of $G_{\text {diff }}$ for $\mathrm{He}, \mathrm{Ar}, \mathrm{Kr}$ at such experimental conditions are calculated using (1) and (2) and they are equal $4.65 \cdot 10^{-17}$, $1.47 \cdot 10^{-16}, 2.13 \cdot 10^{-16} \mathrm{~kg} / \mathrm{s}$, respectively.

\section{Numerical approach}

Now let us provide the main points of the developed model. During the simulation The Monte Carlo test particle method [16] is applied. Each new particle generated at the channel inlet is uniformly distributed over the capillary cross-section. The position of each particle at the channel entrance is simulated by the following way. For a circular inlet with the radius $r$ (see Fig. 4), the number of molecules $d N_{\rho}$ located inside the ring with the radius $\rho$ and the width $d \rho$ is determined as $d N_{\rho}=2 N r d \rho / r^{2}$ based on which the

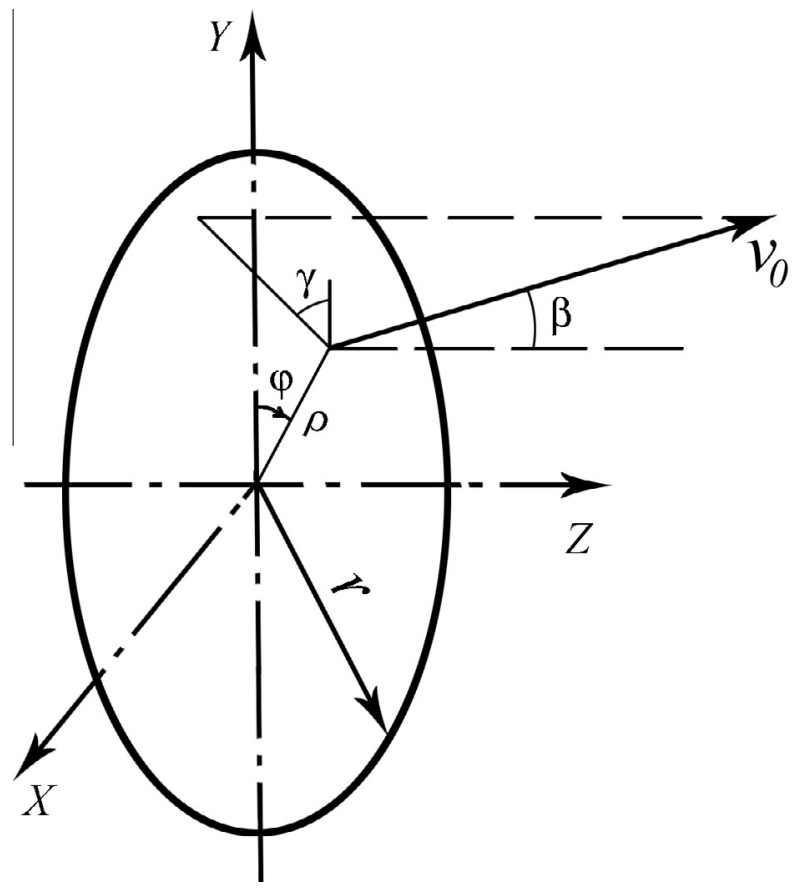

Fig. 4. The initial coordinates of the particle diffusely emitted from the circular cross-section. 
molecule distribution function over the radius is $\eta(\rho)=2 \rho / r^{2}$; the molecule emission radius is obtained from the condition $\int_{0}^{\rho} \eta(\rho) d \rho=R f()$; hence, $\rho=r / \sqrt{R f()}$. Due to the uniform distribution of entering molecules over the channel entrance, the polar angle $\varphi$ can be arbitrary, i.e. $\varphi=2 \pi R f()$. Thus, the initial position of a gas particle at the channel inlet can be written as follows: $x_{0}=\rho \cos (\varphi), y_{0}=\rho \sin (\varphi), z_{0}=0$.

The particles diffusively emitted from the channel inlet surface have the Maxwellian distribution of the molecular velocity at the temperature $T_{g}=300 \mathrm{~K}$. The inner channel surface temperature is $T_{s}=300 \mathrm{~K}$.

The algorithm for simulating the equilibrium components of the particle's velocity is detailed in [16]; therefore, let us give only a pseudo-code here. $\quad v_{z}=v_{m} \sqrt{-\ln (R f())}, \quad a=\sqrt{-\ln (R f())} \gamma=$ $2 \pi R f(), \quad v_{x}=v_{m} a \cos (\gamma), \quad v_{y}=v_{m} a$ sin $(\gamma)$, where $v_{m}=128$, $95 \sqrt{T_{g} / \mu}$ is the most probable velocity of the gas particle at the temperature $T_{g}$, where $\mu$ is the molecular weight of the particle.

The particle movement is simulated until the particle leaves the capillary. It can leave through the same cross section where it was generated or from the opposite section. The ratio of the number particles leaving from the opposite section to the total number of generated particles provides the transmission probability $W$.

When a gas particle passes through the channel, each collision of the atom with the surface is simulated, i.e. the gas atom scattering over the solid body structure is simulated with the transformation of its velocity vector, which is caused by the use of two different coordinate systems. The first system $(X Y Z)$ is linked with the channel; the second one $\left(X^{\prime} Y^{\prime} Z^{\prime}\right)$ is connected with the local collision area, whose normal is perpendicular to the channel axis. The particle continues its movement after interaction with the surface element to the next collision point or to the channel outlet/inlet and has a back-transformed velocity vector. The example of gas particle movement inside the cylindrical channel and gas atom scattering on the local collision area of the channel surface are presented on Fig. 5.

The model of interaction between the gas atom and the channel surface is constructed on the basis of following assumptions: (a) the atoms of a gas and a solid body surface represent spheres with radiuses and masses conforming with the characteristics of the real atoms; (b) the channel surface is represented by a lattice structure producing thermal vibrations at the temperature $T_{s}$. The thermal displacement of solid body atoms from equilibrium position is also considered; (c) the interaction forces between the gas atom and the channel surface are calculated considering all the atoms of the surface structure and the atoms adsorbed thereon. Only the solid body atoms that are at most $10 \AA$ distant from the gas atom are considered; (d) the forces of interaction between the solid body atoms are simulated only between the nearest neighbors; (e) in

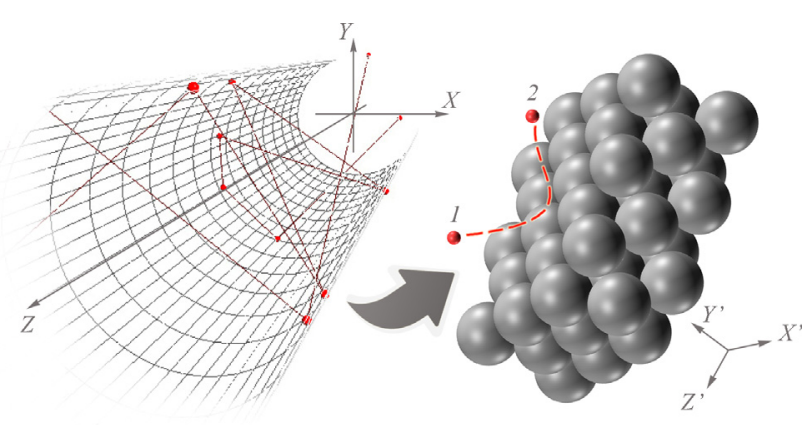

Fig. 5. He atom movement inside the cylindrical channel (left) and $H e$ atom scattering on the local collision area of the channel surface which is presented by $f c c$ layer of $\mathrm{Ag}$ atoms (right): 1,2 - positions of the He atom (before and after collision, respectively). case the kinetic energy of a gas atom is insufficient for overcoming the attractive forces of solid body atoms, then such a gas atom is considered to be adsorbed and is diffusely emitted at the solid body temperature $T_{s}$ and the direction of the emission is independent of the incidence direction; (f) The integration time interval $\Delta t$ is recalculated after each collision of the particle with the surface based on the condition that the gas atom was able to move by 0.05 Å during $\Delta t$.

It was shown [1] that producing thermal vibrations atoms of clean metal surface have a significant influence on the process of gas particles scattering. Therefore, in the case of clean metal surface we introduce the function $\sigma\left(T_{s}\right)$ that allows to randomly set the thermal displacement of metal atoms from their equilibrium positions. This setup process is repeated before the each collision of gas particle with the solid body atoms.

The thermal movement of the solid body atoms is described by a harmonic interaction potential $\boldsymbol{U}(\Delta \boldsymbol{r})=k \Delta \boldsymbol{r}^{2} / 2$, where $k$ is the bonding force constant (it is assumed that the force constants for each direction of the three-dimensional coordinate system are equal), $\Delta \mathbf{r}$ is the vector of atom displacement from the equilibrium position. Thus, taking into account that $\boldsymbol{F}(\boldsymbol{\Delta} r)=-d \boldsymbol{U}(\boldsymbol{\Delta} r) / d \mathbf{r}$, the force acting on the solid body atom displaced from the equilibrium position can be written as follows:

$\mathbf{F}(\Delta r)=-k \Delta r$

The motion equation for a gas atom with the mass $M_{g}$, which interacts with local collision area, is

$\mathbf{R}(\tau)=\mathbf{R}(0)+\dot{\mathbf{R}}(0) \tau-\left(1 / M_{g}\right) \sum_{\mathbf{M}} \int_{0}^{\tau} d s \int_{0}^{s} d u \mathbf{F}_{g s, g a}(\mathbf{M}, u)$,

where $\mathbf{R}(\tau)$ is the position of the gas atom at the moment $\tau ; \mathbf{F}_{g s}$ is the interaction force between the gas atom and the $\mathrm{M}$ solid body atom; $\mathbf{F}_{g a}$ is the interaction force between the gas atom and the atom adsorbed on the surface.

The motion equation of the $\mathbf{N}$ solid body atom with the mass $M_{s}$ is

$\begin{aligned} \mathbf{Y}(\mathbf{N}, \tau)= & \mathbf{Y}_{t}(\mathbf{N}, \tau) \\ & +\left(1 / M_{s}\right)\left[\sum_{\mathbf{M}} \int_{0}^{\tau} d s \int_{0}^{s} d u \mathbf{F}_{s s, s a}(\mathbf{M}, u)+\int_{0}^{\tau} d s \int_{0}^{s} d u \mathbf{F}_{g s}(\mathbf{N}, u)\right]\end{aligned}$

where $\mathbf{Y}(\mathbf{N}, \tau)$ is the displacement of the $\mathbf{N}$ solid body atom at the moment $\tau ; \mathbf{Y}_{t}(\mathbf{N}, \tau)$ is the thermal displacement of the $\mathbf{N}$ atom at the moment $\tau$. The right part of the expression (5) describes the influence of the neighboring $\mathbf{M}$ atoms of the solid body subject to the forces $\mathbf{F}_{s s, s a}$ and the influence of the gas atom on the $\mathbf{N}$ atom subject to the force $\mathbf{F}_{g s}$ at the moment $u$ within the interval $0<u<\tau$.

\section{Results and discussion}

Based on the above described formalism of gas-surface interaction using the computer simulation, an attempt to describe the influence of chemical composition of internal surface of the cylindrical channel on the free-molecular gas flow has been undertaken. Since the data on the interaction potentials between the Ti atom and the noble gas atoms were not present in the available literature, the simulation employed $\mathrm{Ag}$ as the solid body atoms.

During the simulation two cases have been considered. First, we have studied the atomically clean $\mathrm{Ag}$ surface, where each act of collision of a particle with the channel surface represents scattering of the gas atom ( $\mathrm{He}, \mathrm{Ar}, \mathrm{Kr})$ on the face-centered cubic $(f c c)$ structure of silver with the bonding force constant $k_{A g}$. That case corresponds to the fresh deposition of $A g$ on the inner channel surface. In the second case, the gas ( $\mathrm{He}, \mathrm{Ar}, \mathrm{Kr}$ ) particle interacts with the surface, 


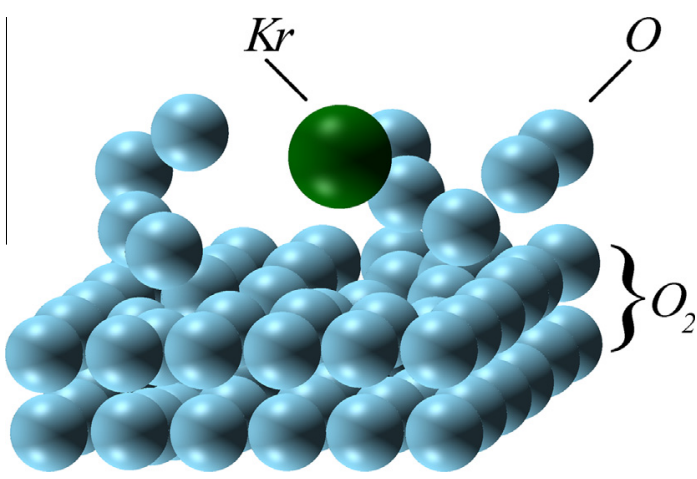

Fig. 6. The example of interaction between $K r$ atom and "dirty" surface, $\theta=0.4$.

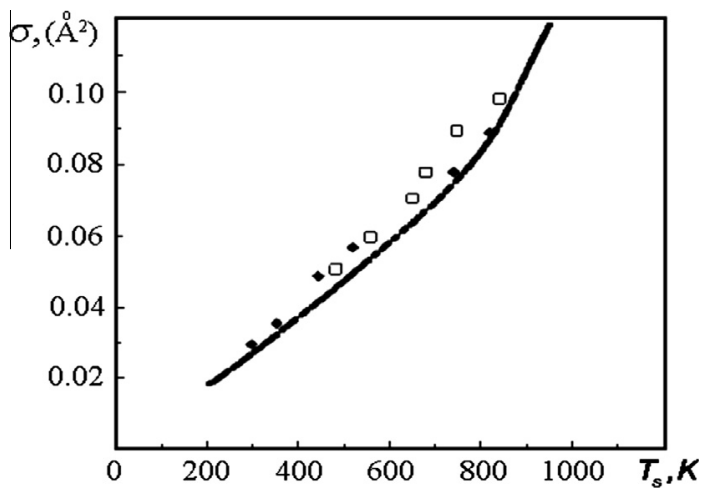

Fig. 7. Root-mean-square thermal displacement $\sigma$ of $\mathrm{Ag}$ atoms from the equilibrium position as a function of the surface temperature $T_{s}[18]$.

which is modeled as a monolayer of vertically oriented $\mathrm{O}_{2}$ molecules with the bonding force constant $k_{O}$. The interatomic distance between oxygen atoms of $\mathrm{O}_{2}$ molecule is $1.2 \AA$. The second layer is physically adsorbed and consists of oxygen atoms with $\theta=R f()$ and representing $\mathrm{H}_{2} \mathrm{O}, \mathrm{CO}, \mathrm{NO}, \mathrm{CO}_{2}$, i.e. the compounds of the spectrum of residual gasses of the vacuum chamber (see Fig. 1). The adsorbed atoms of that layer are bonded to the monolayer of $\mathrm{O}_{2}$ molecules with the bonding force constant $k_{O}+$ Ads. The distance between $\mathrm{O}_{2}$ molecule and corresponding physically adsorbed oxygen atom is $2 \AA$ A. That case conforms with the so-called "dirty" surface (see Fig. 2). There is no need to simulate surface atoms of $A g$ because the obtained Auger spectra fail to "demonstrate" the deposited metal atoms in that case. It means that only adsorbate will influence an incident gas atom. $\theta=R f()$ means that for the current act of collision the fraction of surface covered by adsorbed atoms is randomly selected between 0 and 1 from the uniformly distribution. For example, at $\theta=0$ incident gas particle interacts only with layer of vertically oriented $\mathrm{O}_{2}$ molecules. At $\theta=1$ each $\mathrm{O}_{2}$ molecule is physically bonded with above situated $O$ atom which represents $\mathrm{H}_{2} \mathrm{O}, \mathrm{CO}, \mathrm{NO}, \mathrm{CO}_{2}$.

The example of interaction between $\mathrm{Kr}$ atom and local collision area which is presented by layer of vertically oriented $\mathrm{O}_{2}$ molecules covered with $O$ atoms due to adsorption (case of "dirty" surface) at $\theta=0.4$ is presented on Fig. 6 .

The parameters of the interaction potential for the " $\mathrm{Ag}$ - noble gasses" pairs were taken from [17]. The data on the " $\mathrm{Ag}-\mathrm{Ag}$ " pair, as well as the function $\sigma\left(T_{s}\right)$ (see Fig. 7) describing root-meansquare thermal displacement of $\mathrm{Ag}$ atoms from equilibrium position was taken from [18]. As it can be seen from the Fig. 7 that $\sigma=0,025 \AA^{2}$ at $T_{s}=300 \mathrm{~K}$ for the $\mathrm{Ag}$ atoms. The potential parameters for the "noble gasses - $O$ " pairs are found in [19]. For the reader convenience sake all of the used potentials and their parameters are presented in Appendix 1.

During the simulation the transmission probability $W_{c a l}$ is obtained for two different surface conditions and for the gasses $\mathrm{He}$, $A r, K r$. The simulation results and the experimental data expressed in the form of the relative gas flow rate $G$ are given in Table 1 . The $G$ is related to $W_{\text {cal }}$ in the same manner as the mass flow rate $G_{\text {exp }}$, i.e., $G=W_{\text {cal }} / W_{\text {diff }}$, where $W_{\text {diff }}$ can be calculated using (2).

The notation " $O+A d s$ " applies to the surface of vertically oriented $\mathrm{O}_{2}$ molecules covered with oxygen atoms subject to $\theta=R f()$. " $A g$ " corresponds to a freshly deposited clean silver surface.

According to the data presented in Table 1, the developed model adequately describes only relative gas flows for $\mathrm{Ar}$ and $\mathrm{Kr}$ regardless of the surface conditions. As for $\mathrm{He}$, the calculated gas flow rate for the freshly deposited silver surface sufficiently agrees with the experimental data and disagrees when the channel surface is completely covered with adsorbed atoms. Probably, that the use of a physically and chemically simple model of an adsorbate, when oxygen atoms represent $\mathrm{H}_{2} \mathrm{O}, \mathrm{CO}, \mathrm{NO}, \mathrm{CO}_{2}$, does not allow to obtain good results for the flow of such a light gas as $\mathrm{He}$. While the particles $\mathrm{Ar}$ and $\mathrm{Kr}$ have more chances for penetration into the surface due to their larger weight and more distinct potential well depth, which increases their adsorption probability followed by the relaxation on the surface and further diffusive emission that eventually leads to the decrease of the gas flow through the channel. In our opinion, the use of a more physically and chemically complex adsorbate model, where the interaction with other adsorbed atoms and molecules (see Fig. 1) in addition to the oxygen is simulated as well as the structure of adsorption layers is taken into consideration, would provide good description of the experimental data, including in the case of $\mathrm{He}$.

The choice of the values of $k_{A g}, k_{O}, k_{O}+$ Ads was based on the principle of the best agreement of $G$ with the experimental data. The thus obtained values are given in Table 2.

When analyzing the data from Tables 1 and 2, there is a direct relation observed between the relative gas flow rate and the value of the respective bonding force constant. Such a relation can be explained as follows: with the increase of the value of the bonding force constant characterizing the bonding rigidity between

Table 2

Values of the bonding force constants $k_{A g}, k_{O}$ and $k_{O+A d s}$ for " $A g-A g$ ", " $O+A d s "$ systems, respectively.

\begin{tabular}{lll}
\hline$A g$ & $O+A d s$ & \\
\hline$k_{A g}=285$ & $k_{O}=10$ & $k_{O+A d s}=1$ \\
\hline
\end{tabular}

Table 1

Relative gas flow rate $G$ of the cylindrical channel for gasses $\mathrm{He}, \mathrm{Ar}, \mathrm{Kr}$ and different surface compositions.

\begin{tabular}{|c|c|c|c|c|c|c|}
\hline & \multicolumn{2}{|l|}{$\mathrm{He}$} & \multicolumn{2}{|l|}{$A r$} & \multicolumn{2}{|l|}{$K r$} \\
\hline & Experiment [14] & Simulation & Experiment [14] & Simulation & Experiment [14] & Simulation \\
\hline $\mathrm{Ag}$ & 1.62 & 1.51 & 1.23 & 1.285 & 1.15 & 1.1 \\
\hline$O+A d s$ & 1.04 & 2.1 & 1.01 & 1.0 & 1.00 & 1.0 \\
\hline
\end{tabular}


neighboring atoms, the degree of the interaction elasticity between the incoming gas particle and the surface under consideration is increased. Thus, for $k_{A g}=285$, more elastic interaction between the gas atoms and the surface occurs compared to the cases when $k_{O}=10$ and $k_{O+A d s}=1$. The increase of the interaction rigidity results in the increase of the share of specularly reflected particles, which in turn leads to the increase of the flow rate. The obtained values of the bonding force constants $k_{O}$ and $k_{O+A d s}$ characterize a "softer" surface, whose chemical composition is represented by the adsorption conglomerate based on the oxygen atoms from residual gasses of the vacuum chamber, which considerably influences the flow rate.

\section{Conclusions}

An attempt to describe a rarefied gas flow in the cylindrical channel, whose surface chemical composition conforms with the real experimental conditions, has been undertaken using the developed model. The simulation of the solid body surface structure and the interaction potential between the gas atom and each surface atom, including the adsorbed atoms, as well as the taking into account of the internal interaction between the surface atoms, forms the basis for the gas-surface interaction process. Thermal vibrations of the surface atoms are also considered. In terms of the chemical composition, the atomically clean $\mathrm{Ag}$ surface is changed to the surface fully covered with the adsorbate. The adsorbate is simulated by oxygen molecules and atoms as, according to the data of the Auger-spectroscopic analysis, their primary presence on the surface proves to be true.

Comparison of the obtained data with the data obtained in the controlled-surface experiments shows that a rather good description of the relative gas flow in the channel can be achieved using the developed approach for the clean silver surface as well as in the case when the channel surface is fully covered with the adsorbate atoms. The main cause of the dependence of the gas flow rate on the chemical state of the channel surface lies in the change of the nature of interaction between the gas atom and the surface particles. Thus, for example, specular reflection from the surface element prevails in the case of the clean silver surface, which contributes to the increase of the flow rate. In the case of "dirty" channel surface when it is fully covered with the adsorbate, it leads to the reduction of the share of elastic interaction and consequently to the decrease of flow rate.

The developed model describing the rarefied gas flow in the channel with regard to the chemical composition of its surface can be used for investigation of the processes occurring at the gas-solid body interface. The demonstrated model enables, for example, to predict the mass flow rates in the micro-channels, which is primarily driven by the practical needs. The present study also bears fundamental importance connected with the development of model approximations of the heat-and-mass transfer processes in the "rarefied gas-solid body" system.

\section{Acknowledgements}

The present research was supported by Russian Ministry of Education and Science (ADTP Project NP-45, No.: 2.2.2.2/5579) and American Foundation (CRDF Award No.: RUE1-1516-EK-06).

\section{Appendix 1}

Interaction potential between noble gas atom and $\mathrm{Ag}$ atom [17] $U(x)=\varepsilon\left[\exp ^{2 a\left(r_{0}-x\right)}-2 \exp ^{a\left(r_{0}-x\right)}\right], a=0.75 ;$
$H e: \varepsilon=0.00629(e V), r_{0}=3.25(\AA)$;

$A r: \varepsilon=0.00737(e V), r_{0}=2.18(\AA) ;$

$K r: \varepsilon=0.00933(e V), r_{0}=2.28(\AA)$.

Interaction potential between $\mathrm{Ag}-\mathrm{Ag}$ [18]

$U(x)=\varepsilon_{1} \exp ^{-p\left(x / r_{0}-1\right)}-\varepsilon_{2} \exp ^{-q\left(x / r_{0}-1\right)}$,

$\varepsilon_{1}=0.1028(\mathrm{eV}), \varepsilon_{2}=1.178(\mathrm{eV})$

$p=10.928, q=3.139, r_{0}=2.889(\AA)$.

Interaction potential between noble gas atom and $O$ atom [19]

$U(x)=\varepsilon\left[\exp ^{-2 \beta\left(x / r_{0}-1\right)}-2 \exp ^{-\beta\left(x / r_{0}-1\right)}\right]$,

$H e: \varepsilon=0.0021(\mathrm{eV}), \beta=6.5, r_{0}=3.27(\AA)$;

$A r: \varepsilon=0.0078(e V), \beta=6.2, r_{0}=3.6(\AA) ;$

$K r: \varepsilon=0.0093(e V), \beta=6.2, r_{0}=3.75(\AA)$.

\section{References}

[1] F. Goodman, H. Wachman, Dynamics of Gas-Surface Scattering, Academic Press, New York, 1976. p. 327.

[2] M. Kaminsky, Atomic and Ionic Impact Phenomena on Metal Surfaces, Springer, Berlin, 1965. p. 402.

[3] L. Thomas, Rarefied Gas Dynamics, Academic Press, New York, 1967. p. 155.

[4] E. Shteiheil, W. Scherber, M. Seide, H. Reiger, Investigation on the interaction of gases and well defined surfaces with respect to possibilities for reduction of aerodynamic friction and aerothermal heating, in: J.L. Potter (Ed.), Rarefied Gas Dynamics, Acad. Press, New York, 1977, pp. 589-602.

[5] J. Maxwell, The Scientific Papers of James Clerk Maxwell, Dover, New York, 1890.

[6] C. Cercignani, M. Lampis, Kinetic models for gas-surface interactions, Transport Theor. Stat. Phys. 1 (1971) 101-114.

[7] M. Epstein, A model of the wall boundary condition in kinetic theory, J. AIAA 5 (10) (1967) 1797-1800.

[8] H. Wachman, I. Greber, G. Kass, Molecular dynamics computations of scattering from a surface using a Lennard-Jones model of a solid, in: A.R. Seebass (Ed.), Progress in Astronautics and Aeronautics, in: B.D. Shizgal, D.P. Weaver (Eds.), Rarefied Gas Dynamics, vol. 158, AIAA, Washington, DC, 1993, p. 461.

[9] M. Cieplak, J. Koplik, J.R. Banavar, Applications of statistical mechanics in subcontinuum fluid dynamics, Physica A 274 (1999) 281-293.

[10] S.V. Nedea, A.J.H. Frijns, A.A. van Steenhoven, A.J. Markvoor, P.A.J. Hilbers, Hybrid method coupling molecular dynamics and Monte Carlo simulations to study the properties of gases in microchannels and nanochannels, Phys. Rev. E 72 (2005) 016705

[11] K. Yamamoto, H. Takeuchi, T. Hyakutake, Characteristics of reflected gas molecules at a solid surface, Phys. Fluids 18 (2006) 046103.

[12] S.V. Nedea, A.J. Markvoort, A.A. van Steenhoven, P.A.J. Hilbers, Heat transfer predictions for micro-/nanochannels at the atomistic level using combined molecular dynamics and Monte Carlo techniques, J. Heat Transfer 131 (3) (2009) 033104. 8 pages.

[13] Van den Akker, A.J.H. Frijns, P. Hilbers, A.A. van Steenhoven, A molecular dynamics boundary condition for heat exchange between walls and a fluid, Mol. Simul. 37 (10) (2011) 855-864.

[14] O.V. Sazhin, S.F. Borisov, Influence of the state of a surface on the formation of a rarefied gas flow in a channel, J. Eng. Phys. Thermophys. 74 (5) (2001) 12321238.

[15] F. Sharipov, V. Seleznev, J. Phys. Chem. Ref. Data 27 (1998) 657-706.

[16] G.A. Bird, Molecular Gas Dynamics and the Direct Simulation of Gas Flows, Oxford University Press, 1994.

[17] S. Ossicini, Interaction potential between rare-gas atoms and metal surfaces, Phys. Rev. B. 33 (2) (1986) 873-878.

[18] F. Cleri, V. Rosato, Tight-binding potentials for transition metals and alloys, Phys. Rev. B. 48 (1) (1993) 22-33.

[19] V. Aquilanti, R. Candori, F. Pirani, Molecular beam studies of weak interactions for open-shell systems: the ground and lowest excited states of rare gas oxides, J. Chem. Phys. 89 (10) (1988) 6157-6164. 